\title{
Implementation of intensity-modulated radiation therapy in the treatment of carcinoma of the nasopharynx and oropharynx: A retrospective single-institution analysis
}

\author{
Emile S. Gogineni', Jason D. Kehrer², Guy C. Jones, ${ }^{2, *}$, Bryan Adams ${ }^{3}$ and Rex Kiteley ${ }^{3}$ \\ 1 New York Institute of Technology, College of Osteopathic Medicine, Old Westbury, NY, USA \\ 2 Radiation Oncology Branch, National Cancer Institute, National Institutes of Health, Bethesda, MD, USA \\ ${ }^{3}$ Department of Radiation Oncology, Walter Reed National Military Medical Center, Bethesda, MD, USA
}

\begin{abstract}
Background: Intensity-modulated radiation therapy (IMRT) has become the standard of care for the treatment of nasopharyngeal and oropharyngeal carcinoma. As the majority of head and neck IMRT is performed in community cancer centers, which often have lower volume than major academics centers, it is important to ensure that cancer control rates and patient survival are comparable between community treatment centers and large academic institutions with site-specific oncologists. Material and methods: From February 2003 to August 2008, 8 patients with nasopharyngeal carcinoma and 42 patients with oropharyngeal carcinoma were treated with IMRT at the National Naval Medical Center in Bethesda, Maryland. Results: At a median follow-up of 61 months, the overall survival (OS) was $89 \%$ and local control (LC) was $94 \%$ for all patients treated. Patients with nasopharyngeal cancer achieved $100 \%$ LC and a 5-year OS of $100 \%$ with a median follow-up of 64.5 months. Patients with oropharyngeal cancer achieved 93\% LC (median time to loco-regional recurrence of 11 months) and an OS of $88 \%$ at a median follow-up of 58 months. All patients with LC at 18 months remained free of loco-regional failure throughout the follow-up period. Conclusions: Institutional reviews from large academic centers provide encouraging results regarding the efficacy and delivery of IMRT in the treatment of head and neck cancer. This retrospective, single-institution analysis demonstrates the ability to effectively implement and utilize IMRT in the treatment of head and neck cancer in an environment with relatively modest patient volume. The excellent OS and LC outcomes at our institution confirm the potential for smaller academic and community centers with lower head and neck patient volume to deliver effective therapy despite the inherent complexity of IMRT.
\end{abstract}

Keywords: head and neck; IMRT; oropharynx; nasopharynx

\section{Introduction}

External beam radiation therapy (EBRT) plays an integral role in the treatment of head and neck (H\&N) cancer in both the definitive and adjuvant settings. In recent years, the techniques used to deliver EBRT for head and neck cancer have shifted from 3-dimensional conformal RT (3DCRT) to intensity-modulated radiation therapy (IMRT) with image-guided radiation therapy (IGRT).

This transition of techniques has been rapidly adopted by nearly every radiation treatment center in the United States, following studies from large academic centers demonstrating clinical and dosimetric superiority and diminished adverse effects [1, 2]. The clinical adoption of IMRT demands an exceptional understanding of radiographic anatomy as well as a strong knowledge of oncological considerations, including patterns of disease spread and recurrence. Thus, as the use of IMRT for H\&N cancer has become more prevalent, experts have sought to provide aid to smaller centers adopting these techniques in the form of guidelines for volume delineation [3-5] and contouring atlases [6].
Still, concerns continue to exist about the ability of smaller institutions to match the clinical outcomes of highervolume centers using newer treatment modalities. Many radiation oncologists in smaller community centers have trained at centers with a high-volume of $\mathrm{H} \& \mathrm{~N}$ cancers in the IMRT era and thus have become proficient in these techniques. However, at centers with low patient volume of $\mathrm{H} \& \mathrm{~N}$ cancer, lack of ongoing utilization of these techniques

*Corresponding author: Guy C. Jones, M.D., Radiation Oncology Branch, National Cancer Institute, National Institutes of Health, 9000 Rockville Pike, Bethesda, MD 20892, USA. Tel.: (301) 496-5457; Email: guy.jones@nih.gov

Received 13 November 2015 Revised 11 January 2015 Accepted 20 January 2015 Published 28 January 2016

Citation: Gogineni ES, Kehrer JD, Jones GC, Adams B, Kiteley R. Implementation of intensity-modulated radiation therapy in the treatment of carcinoma of the nasopharynx and oropharynx: A retrospective singleinstitution analysis. J Clin Radiat Oncol. 2016; 1(1):1-6. DOI:10.14312/23978511.2016-1

Copyright: (c) 2016 Gogineni ES, et al. Published by NobleResearch Publishers. This is an open-access article distributed under the terms of the Creative Commons Attribution License, which permits unrestricted use, distribution and reproduction in any medium, provided the original author and source are credited. 
may degrade proficiency in that area. In addition, these techniques change over time and require physicians to keep abreast of the latest developments in these methods. Given that these concerns exist, our institution sought to review our own experience and outcomes with regard to IMRT for head and neck cancer at our lower volume center, in order to verify that we are providing our patients with the highest quality of care.

\section{Material and methods}

From February 2003 to August 2008, 50 patients with nasopharyngeal $(n=8)$ or oropharyngeal $(n=42)$ carcinoma were treated with intensity-modulated radiation therapy (IMRT) at the National Naval Medical Center in Bethesda, Maryland. Of the 50 patients, 45 were male and 5 were female. All patients received treatment for squamous cell carcinoma $(n=49)$ with the exception of one patient with adenoid cystic carcinoma of the oropharynx. One patient treated for nasopharyngeal squamous cell carcinoma was lost to follow-up after 7 months and thus was excluded from this analysis.

All oropharyngeal primary cancers arose from the tonsil or base of tongue. Fourteen of the 42 patients (33\%) with oropharyngeal cancer and 3 of the 7 (43\%) patients with nasopharyngeal cancer had a known history of smoking tobacco products. All nasopharyngeal cancer patients presented with diseases at stages II (5/7, 71\%) and III (2/7, 29\%). Oropharyngeal cancer patients presented at stages I-IVC, with 31/42 (74\%) being IVA. Two patients with oropharyngeal cancer had distant metastases (Stage IVC) at the time of diagnosis but were still treated definitely. These patients were excluded from our data analysis. Patient demographics are summarized in Table 1.

Our center is a stand-alone radiation oncology practice and all plans were generated on-site by in-house physicists and dosimetrists. Several IMRT techniques were employed with selection based upon physician preference, dosimetric advantages, and machine time availability. A Corvus planning system was used to design IMRT plans using fixed beam (6-9 fields), volumetric modulated arc therapy (4-7 arcs), or a combination of the two techniques. Patients received $6800-7200$ cGy in 180 cGy to 200 cGy daily fractions. A low anterior neck field was matched in 46 of the 49 cases (94\%) to deliver 4500-5400 cGy. Treatments were delivered using a Clinac CL21EX and/or CL600C. Figure 1 shows representative treatment plans for an oropharyngeal and a nasopharyngeal cancer utilizing fixed beam IMRT (7 fields) and volumetric modulated arc therapy (7 arcs), respectively. Dosimetric data, including dose received by the mandible, parotid gland, larynx, spinal cord and brainstem is summarized in Table 2. All hot spots were kept under $115 \%$ of the prescribed dose and were never located within organs at risk. Constraining of the oral cavity and constrictors was not standard at our institution during the period this data was collected.

Of the 50 patients that were treated, 49 were treated with definitive IMRT and 1 received re-treatment for recurrent base of tongue squamous cell carcinoma. No patient underwent an oncologic surgery before radiotherapy. Five of the 42 patients (12\%) treated for oropharyngeal
Table 1 Patient characteristics ( $n=47)$.

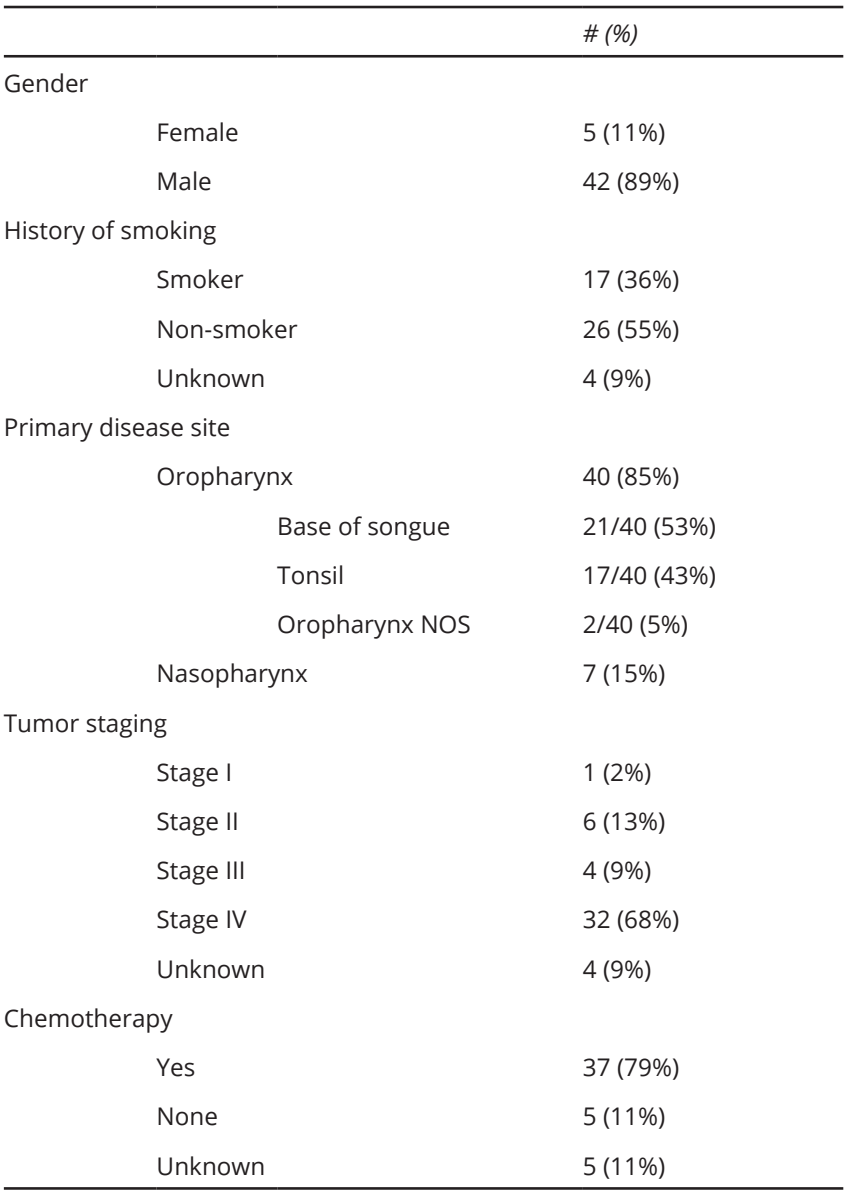

Abbreviations: NOS = not otherwise specified.

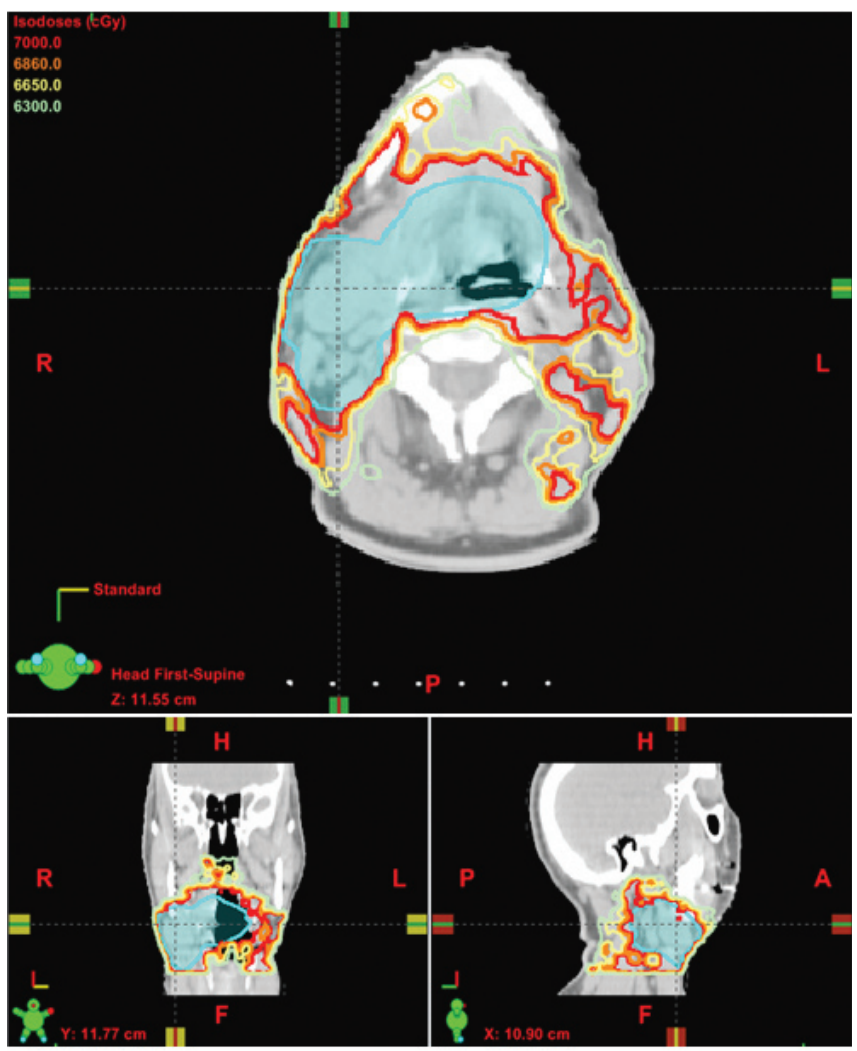

Figure 1a IMRT planning image for a patient with oropharyngeal carcinoma. $100 \%, 98 \%, 95 \%$, and $90 \%$ isodose lines are displayed as red, orange, yellow, and green respectively. Planning Target Volume (PTV) in displayed in blue. 


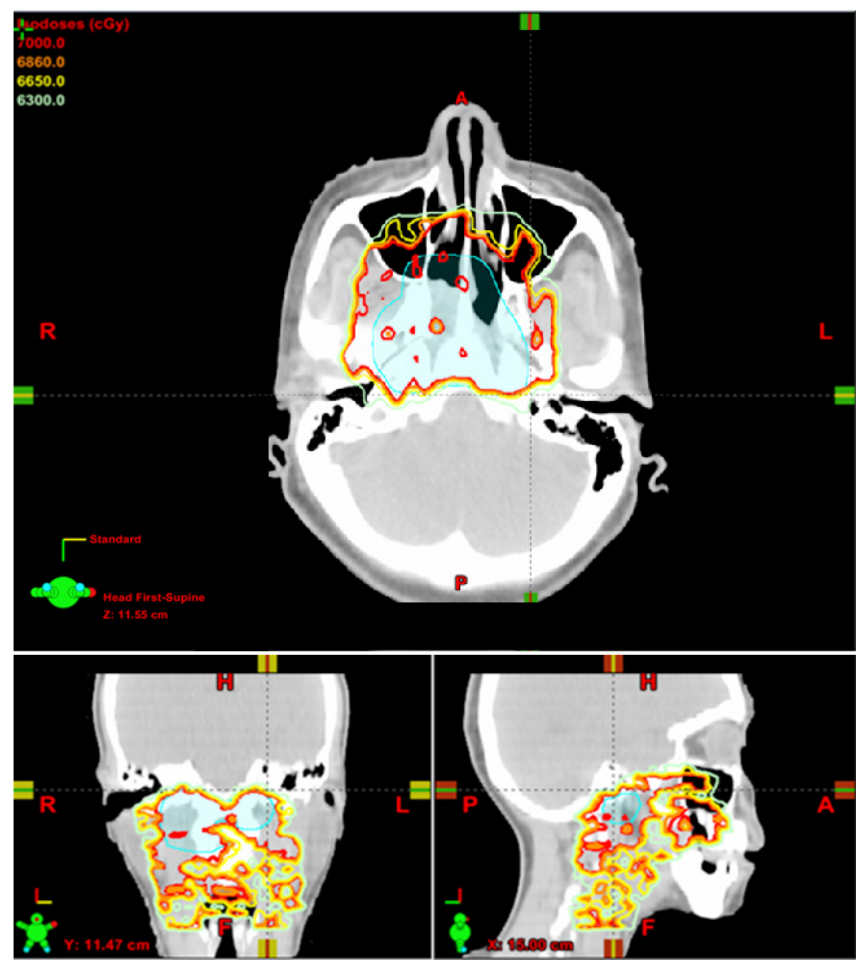

Figure 1b IMRT planning image for a patient with nasopharyngeal carcinoma. $100 \%, 98 \%, 95 \%$, and $90 \%$ isodose lines are displayed as red, displayed in blue.

Table 2 Summary of all dosimetric data. orange, yellow, and green respectively. Planning Target Volume (PTV) in

cancer received IMRT alone and the remainder received concurrent chemoradiation with a two drug combination of either cisplatin or carboplatin and either 5-Flourouracil (5FU) or cetuximab. One patient received one cycle of neoadjuvant treatment with TPF chemotherapy (docetaxel, cisplatin, and 5FU) prior to receiving definitive concurrent chemoradiation with cisplatin. Two of the 7 patients (29\%) treated for nasopharygeal cancer received IMRT alone and the remainder received concurrent cisplatin followed by adjuvant carboplatin and 5FU or carboplatin and docetaxol.

\section{Results}

At a combined median follow-up time of 61 months (range: 7-102 months), all patients had an overall survival (OS) and local control (LC) of 89\% (42/47 patients) and 94\% (44/47 patients), respectively. When looking specifically at patients treated for nasopharyngeal primary cancers, there was a $100 \%$ (7/7 patients) rate of LC and a 100\% (7/7 patients) OS at a median follow-up of 64.5 months (range: 62-95 months). For patients treated for oropharyngeal primary cancers, there was a 93\% (37/40 patients) rate of LC and an $88 \%$ (35/40 patients) OS at a median follow-up of 58 months (range, 7-102 months). The median time to locoregional recurrence was 11 months (range: 7-18 months). Patient outcomes are summarized in Table 3 and Figure 2.

\begin{tabular}{|c|c|c|c|c|c|c|c|}
\hline Site & & $\begin{array}{l}\text { PTV (\% of target } \\
\text { that received } \\
100 \% \text { of dose) }\end{array}$ & $\begin{array}{c}\text { Max mandible } \\
\text { dose (Gy) }\end{array}$ & $\begin{array}{c}\text { Mean } C / L \\
\text { parotid dose (Gy) }\end{array}$ & $\begin{array}{c}\text { Max/Mean } \\
\text { larynx dose (Gy) }\end{array}$ & $\begin{array}{c}\text { Max spinal cord } \\
\text { dose (Gy) }\end{array}$ & $\begin{array}{c}\text { Max brainstem } \\
\text { dose (Gy) }\end{array}$ \\
\hline \multirow[t]{2}{*}{ Nasopharynx } & Mean & 97 & 69 & 23 & $38 / 24$ & 42 & 47 \\
\hline & Median & 96 & 72 & 23 & $38 / 24$ & 43 & 46 \\
\hline \multirow[t]{2}{*}{ Oropharynx } & Mean & 97 & 73 & 21 & $30 / 19$ & 40 & 43 \\
\hline & Median & 97 & 73 & 22 & $38 / 22$ & 43 & 46 \\
\hline
\end{tabular}

Table 3 Patient outcomes at a median followup of 61 months $(n=47)$.

\begin{tabular}{ccc}
\hline & Number & $\%$ \\
\hline 5-year local control & & \\
Combined & $44 / 47$ & 94 \\
Oropharynx & $37 / 40$ & 93 \\
Nasopharynx & $7 / 7$ & 100 \\
5-year overall survival & & \\
Combined & $42 / 47$ & \\
Oropharynx & $35 / 40$ & 88 \\
Nasopharynx & $7 / 7$ & 100 \\
\hline
\end{tabular}

All patients who maintained LC at 18 months remained free of loco-regional failure throughout their follow-up period. Two of the 8 deceased patients (25\%) experienced a loss of local control with three (38\%) developing metastatic disease prior to the time of death. Only one patient (13\%) experienced both loco-regional recurrence and distant metastatic disease.
The majority of patients $(30 / 47,64 \%)$ reported no major (grade $3+$ ) toxicities from treatment. Of those patients who did experience adverse effects, the most common was the need for a long-term percutaneous endoscopic gastrostomy (PEG) tube, ranging from 3 months to 5 years. Other rare toxicities included trismus, stomatitis of the lips and mouth, and dental effects, such as carries, tooth necrosis, bridge deterioration, radionecrosis, and one episode of an exposed mandible. One patient was affected by an esophageal stricture, while three patients experienced hypothyroidism. One patient experienced nerve damage, including spinal accessory nerve toxicity and winged scapula due to long thoracic nerve injury. A summary of all toxicities are shown in Table 4.

\section{Discussion}

Institutional reviews from large academic centers provide encouraging results regarding the efficacy and delivery of IMRT in the treatment of head and neck cancer. IMRT has been shown to be superior to other forms of EBRT in the treatment of head and neck cancer in terms of minimizing 

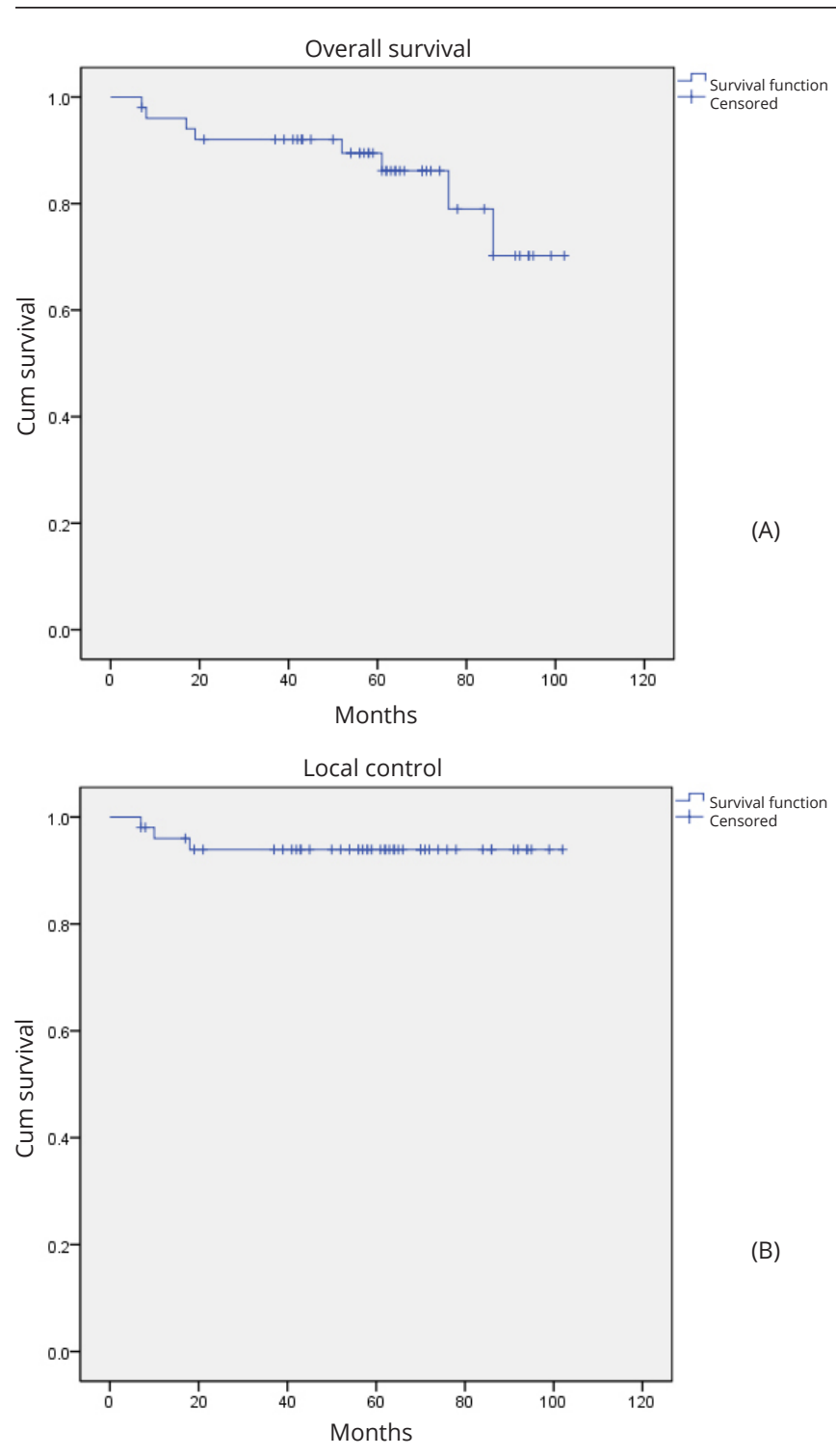

Figure 2A, B Patient outcomes at a median follow-up.

Table 4 Toxicities.

Nasopharynx $(n=7)$

No grade $2+$ toxicities

Nerve damage (CN XI \& long thoracic)

$1(14 \%)$

Oropharynx $(n=40)$

No grade $2+$ toxicities

Acute toxicities

Grade 2 dysphagia

Grade 3 upper GI (PEG tube)

Grade 3 esophageal stricture

Dental complications

Late toxicities

Grade 4 bone (osteoradionecrosis)

Hypothyroid adverse effects without compromising target fields of radiation coverage [7-9]. Numerous studies have explored the incidence of adverse treatment-related effects of EBRT in the IMRT era, including xerostomia and quality of life during and after treatment with the majority showing reduced toxicity [10-13], and these reports have led to IMRT being adopted as the standard of care at radiation oncology institutions across the country.

As the majority of treatment in the United States takes place outside of large institutions, it is important to validate that the beneficial effects of this superior treatment is maintained at smaller academic and community centers. Our staff of 3 physicians treated an average of 9 patients per year for H\&N cancer. This represents approximately $2 \%$ of the 435 new cases per year that were treated at our center. During that same time period, IMRT was routinely utilized for central nervous system and genitourinary cancers, which represented approximately 35\% of cases seen. The purpose of this study was to ensure that our lower volume radiation treatment center had the expertise to achieve results comparable to larger volume centers for the IMRT treatment of H\&N cancers.

Our disease control and survival outcomes compare favourably to several published clinical trials for the radiation treatment of $\mathrm{H} \& \mathrm{~N}$ cancer. Al-Sarraf et al. reported on 147 patients with nasopharyngeal carcinoma treated with concurrent cisplatin and adjuvant cisplatin and 5FU which resulted in a $67 \%$ OS at 5 years after treatment [14]. RTOG 0129 reported on 323 patients with oropharyngeal cancer treated with concurrent cisplatin which resulted in a 73\% OS at 3 years [15]. GORTEC 9401 reported on 109 patients with oropharyngeal cancer treated with concurrent carboplatin and 5FU, which resulted in a 51\% OS at 3 years [16]. The comparison of our data with similar subsets of patients from other trials is shown in Table 5.

When comparing the rate of toxicities seen in our data to results from similar patient subsets, we saw similar results. In our patients, grade $2+$ toxicities were seen in $14 \%$ of nasopharyngeal cases and $40 \%$ of oropharyngeal cases. Kam et al. showed the incidence of grade 2-4 toxicity in patients with nasopharyngeal cancer treated with IMRT to be 39\% [17]. Jilani et al. had an 96\% incidence of grade 2-3 toxicity in retrospective analysis of locally advanced head and neck cancers treated with IMRT [13].

As a military medical center, it is our duty and obligation to provide the highest possible standard of care to our active duty military, retirees, and their dependents. Fortunately, the outcomes of this study were highly encouraging, demonstrating that the local control and survival data from our patients were at least on-par with national trial results including participating institutions from across the country. The excellent LC and OS outcomes of our patients confirm the possibility for centers with low $\mathrm{H} \& \mathrm{~N}$ patient volume to deliver effective therapy despite the inherent complexity of IMRT.

While these results are heartening, certain potential criticisms must be addressed. Although essential to this study, the low volume of patients treated could also be 
Table 5 Data comparison.

\begin{tabular}{|c|c|c|c|c|c|}
\hline \multirow{2}{*}{ Study } & \multirow{2}{*}{$\begin{array}{c}\text { Length of Follow- } \\
\text { up (yrs) }\end{array}$} & \multicolumn{2}{|c|}{ Nasopharynx } & \multicolumn{2}{|c|}{ Oropharynx } \\
\hline & & $L C$ & OS & $L C$ & OS \\
\hline Gogineni, JCRO 2015 & 5 & $7 / 7(100 \%)$ & $7 / 7(100 \%)$ & $37 / 40(93 \%)$ & $35 / 40(88 \%)$ \\
\hline Al-Sarraf, JCO 1998 & 3 & $60 / 78(78 \%)$ & $59 / 78(76 \%)$ & & \\
\hline Bonner (OPC Cetuximab arm), Lancet 2010 & 5 & & & & $96 / 211(46 \%)$ \\
\hline RTOG 0129, NEJM 2010 & 3 & & & $253 / 323(78 \%)$ & $237 / 323(73 \%)$ \\
\hline GORTEC 9401, JNCI 1999 & 3 & & & $72 / 109(66 \%)$ & $56 / 109(51 \%)$ \\
\hline
\end{tabular}

seen as a criticism of this analysis given that it reduces our ability to draw conclusions with strong statistical power. Similarly, as with any single institutional experience, it could be argued that the favourable outcomes seen in this report are a reflection of a more favourable patient population with better overall health and better access to care. Given that the stages of disease seen in our cohort are similar to the national demographic with the majority of patients having locally advanced disease, this is unlikely to be a major factor $[18,19]$.

Another potential confounder in this analysis is the effect of WHO histological subtype of nasopharyngeal cancers and the HPV status of the oropharyngeal cancers in our patient population. Although not an ideal surrogate, the incidence of smoking in this patient population (33\% with oropharyngeal cancer and 43\% with nasopharyngeal cancer) may indicate an overall greater proportion of WHO II/III and HPV+ subtypes which portend a better overall prognosis. Unfortunately, HPV testing was not standard at our institution at the time these patients were diagnosed.

It should also be noted that most of the physicians involved in the treatment planning in this study received their residency training at institutions with high $\mathrm{H} \& \mathrm{~N}$ cancer case volume. It is unclear how this affected the quality of treatment patients received at our center.

Although a number of IMRT techniques and technologies were used in this study, it remains unclear if any particular approach is more efficacious than another. A study by Qi et al., comparing Tomotherapy, True Beam using fixedfield IMRT or rapid arc, and Siemens Oncor, showed that all techniques yielded similar target coverage, but differed in terms of maximum spinal cord dose. C-arm linear accelerator-based IMRT and rapid arc plans were shown to generally produce higher parotid doses, while Tomotherapy cases had the best cord and other organ sparing while minimizing variations of target coverage [20]. Thus, it is certainly possible that the radiation equipment and technologies used at our center could be another confounding source. However the heterogeneity of equipment used to deliver radiation therapy to patients on the Bonner [21], RTOG 0129 [15], GORTEC 9401 [16], and Al-Sarraf trials [14], with which our data were compared, also varied between institutions so the comparisons are likely valid.

\section{Conclusion}

Upfront surgery is an emerging trend in the treatment of oropharyngeal cancer. Patients in this report did not undergo surgery as part of their treatment, and were instead treated with either radiation alone or concurrent chemoradiation. The inclusion of surgery as a component of therapy provides an additional layer of complexity to the treatment course, and further comparison studies will be required to make conclusions regarding community radiation oncology outcomes in the adjuvant setting. The work described in this report was performed to validate our techniques and verify that we are providing our patients the same level of care they would receive from any large academic institution with a higher volume of cases. This retrospective, single-institution analysis demonstrates the ability to effectively implement and utilize IMRT in the treatment of $\mathrm{H} \& \mathrm{~N}$ cancer in an environment with relatively modest patient volume.

\section{Financial Support}

This work was supported by the intramural research program of the National Institutes of Health (NIH), National Cancer Institute, Center for Cancer Research, and the Walter Reed National Military Medical Center.

\section{Disclaimer}

The views expressed in this article are those of the author and do not reflect the official policy of the United States Government.

\section{Conflicts of interest}

The authors have stated that they have no conflicts of interest.

\section{References}

[1] Lee N, Harris J, Garden AS, Straube W, Glisson B, et al. Intensitymodulated radiation therapy with or without chemotherapy for nasopharyngeal carcinoma: Radiation therapy oncology group phase II trial 0225. J Clin Oncol. 2009; 27(22):3684-3690.

[2] Lin A, Kim HM, Terrell JE, Dawson LA, Ship JA, et al. Quality of life after parotid-sparing IMRT for head-and-neck cancer: a prospective longitudinal study. Int J Radiat Oncol Biol Phys. 2003; 57(1):61-70.

[3] Popovtzer A, Ibrahim M, Tatro D, Feng FY, Ten Haken RK, et al. MRI to delineate the gross tumor volume of nasopharyngeal cancers: Which sequences and planes should be used? Radiol Oncolo. 2014; 48(3):323-330. 
[4] Eisbruch A, Marsh LH, Dawson LA, Bradford CR, Teknos TN, et al. Recurrences near base of skull after imrt for head-and-neck cancer: Implications for target delineation in high neck and for parotid gland sparing. Int J Radiat Oncol Biol Phys. 2004; 59(1):28-42.

[5] Eisbruch A, Schwartz M, Rasch C, Vineberg K, Damen E, et al. Dysphagia and aspiration after chemoradiotherapy for head-and-neck cancer: which anatomic structures are affected and can they be spared by IMRT? Int J Radiat Oncol Biol Phys. 2004; 60(5):1425-1439.

[6] Gregoire V, Ang K, Budach W, Grau C, Hamoir M, et al. Delineation of the neck node levels for head and neck tumors: a 2013 update. DAHANCA, EORTC, HKNPCSG, NCIC CTG, NCRI, RTOG, TROG consensus guidelines. Radiother Oncol. 2014; 110(1):172-181.

[7] Lee N, Xia P, Quivey JM, Sultanem K, Poon I, et al. Intensity-modulated radiotherapy in the treatment of nasopharyngeal carcinoma: An update of the ucsf experience. Int J Radiat Oncol Biol Phys. 2002; 53(1):12-22.

[8] Lin S, Pan J, Han L, Guo Q, Hu C, et al. Update report of nasopharyngeal carcinoma treated with reduced-volume intensity-modulated radiation therapy and hypothesis of the optimal margin. Radiother Oncol. 2014; 110(3):385-389.

[9] Ng WT, Lee MC, Hung WM, Choi CW, Lee KC, et al. Clinical outcomes and patterns of failure after intensity-modulated radiotherapy for nasopharyngeal carcinoma. Int J Radiat Oncol Biol Phys. 2011; 79(2):420-428.

[10] Tribius S, Raguse M, Voigt C, Munscher A, Grobe A, et al. Residual deficits in quality of life one year after intensity-modulated radiotherapy for patients with locally advanced head and neck cancer. 2015; 191(6):501510.

[11] Vainshtein JM, Moon DH, Feng FY, Chepeha DB, Eisbruch A, et al. Long-term quality of life after swallowing and salivary-sparing chemo-intensity modulated radiation therapy in survivors of human papillomavirus-related oropharyngeal cancer. Int J Radiat Oncol Biol Phys. 2015; 91(5):925-933.

[12] Lee DS, Kim YS, Cheon JS, Song JH, Son SH, et al. Long-term outcome and toxicity of hypofractionated stereotactic body radiotherapy as a boost treatment for head and neck cancer: The importance of boost volume assessment. Radiat Oncol. 2012; 7:85.

[13] Jilani OK, Singh P, Wernicke AG, Kutler DI, Kuhel W, et al. Radiation therapy is well tolerated and produces excellent control rates in elderly patients with locally advanced head and neck cancers. J Geriatr Oncol. 2012; 3(4).

[14] Al-SarrafM, LeBlanc M, Giri PG, Fu KK, CooperJ, et al. Chemoradiotherapy versus radiotherapy in patients with advanced nasopharyngeal cancer: Phase III randomized intergroup study 0099. J Clin Oncol. 1998; 16(4):1310-1317.

[15] Ang KK, Harris J, Wheeler R, Weber R, Rosenthal DI, et al. Human papillomavirus and survival of patients with oropharyngeal cancer. $\mathrm{N}$ Engl J Med. 2010; 363(1):24-35.

[16] Calais G, Alfonsi M, Bardet E, Sire C, Germain T, et al. Stage III and IV cancers of the oropharynx: Results of a randomized study of gortec comparing radiotherapy alone with concomitant chemotherapy. Bull Cancer. 2000; 87 Spec No:48-53.

[17] Kam MK, Leung SF, Zee B, Chau RM, Suen J, et al. Prospective randomized study of intensity-modulated radiotherapy on salivary gland function in early-stage nasopharyngeal carcinoma patients. J Clin Oncol. 2007; 25(31):4873-4879.

[18] Torre LA, Bray F, Siegel RL, Ferlay J, Lortet-Tieulent J, et al. Global cancer statistics, 2012. CA: a cancer journal for clinicians. 2015; 65(2):87-108.

[19] Jemal A, Siegel R, Xu J, Ward E. Cancer statistics, 2010. CA: a cancer journal for clinicians. 2010; 60(5):277-300.

[20] Qi XS, Ruan D, Lee SP, Pham A, Kupelian P, et al. Dependence of achievable plan quality on treatment technique and planning goal refinement: A head-and-neck intensity modulated radiation therapy application. Int J Radiat Oncol Biol Phys. 2015; 91(4):817-824.

[21] Bonner JA, Harari PM, Giralt J, Azarnia N, Shin DM, et al. Radiotherapy plus cetuximab for squamous-cell carcinoma of the head and neck. $N$ Engl J Med. 2006; 354(6):567-578. 